\title{
Rendimiento lector en estudiantes con trastorno específico de lenguaje
}

\section{Reading performance of students with specific language impairment}

\section{Diana Lafont \\ Pontifica Universidad Católica de \\ Chile}

\section{RESUMEN}

El objetivo de este estudio es proporcionar información sobre el rendimiento lector de 24 estudiantes de 40 año de educación básica, que presentan diagnóstico de Trastorno Específico del Lenguaje (TEL) y que asisten a escuelas públicas y particulares subvencionadas en Santiago de Chile. Se evaluó de manera individual decodificación y comprensión lectora en estos estudiantes y también en un grupo control compuesto por escolares con Desarrollo Típico del Lenguaje (DTL). Los resultados muestran que los estudiantes con TEL poseen diferencias de rendimiento significativas en los promedios obtenidos en las pruebas de comprensión lectora, en comparación al grupo DTL. Este bajo rendimiento no se explica por dificultades en decodificación, ya que en este proceso los promedios alcanzados fueron similares en ambos grupos. Además, a partir del análisis individual de los casos que componen el grupo TEL, se identifica alta variabilidad en sus perfiles lectores. Igualmente, se observa ausencia de correlación entre ambos procesos de lectura en los niños con este déficit lingüístico. Se discuten resultados a partir de le evidencia recogida en el área y desde la Visión Simple de la Lectura (VSL).

Palabras clave: trastorno específico del lenguaje, comprensión lectora y decodificación.

\section{ABSTRACT}

The purpose of this study is to provide information about the reading performance of 24 th graders with have Specific Language Impairment (SLI) attending public and private subsidized schools in Santiago, Chile. Specifically, decoding and reading comprehension were assessed for all students (children with SLI and typical children -TLD- from a contrast group). The results show that students with SLI have significant performance differences on reading comprehension tests when compared to TLD group. Low performance is not explained by difficulties in decoding, since means for decoding are similar in both groups. Also, by means of an individual analysis of data from children with SLI, it was possible to identify a high diversity in their reading profile. There was no correlation between both reading processes among children with SLI. Results are discussed based on the evidence collected in the area and from the Simple Reading Vision (SRV).

FE DE ERRATA: se corrige parte de la introducción. Antes se señalaba "[Desde el año 2006, la Escuela de Fonoaudiología] EI TEL se define...". Enunciado eliminado.
Keywords: specific language impairment, reading comprehension and recoding.
Contacto con el autor: Diana Lafont Santiago - Chile Correo-e: dilafont@uc.cl

Recibido: 01/08/2018 Aceptado: 19/10/2018 


\section{Introducción}

El TEL se define como un déficit en el desarrollo del lenguaje en el que se excluye cualquier tipo de discapacidad que pueda estar interfiriendo en el aprendizaje lingüístico (Bishop \& Snowling, 2004). Este déficit significativo impacta tanto en la esfera receptiva como expresiva de los diferentes componentes del lenguaje, presentándose en formas y grados diversos. Estas características son observables desde los primeros años de vida, lo que se traduce en una presentación tardía de los distintos hitos del desarrollo lingüístico. Los problemas que conlleva el diagnóstico abarcan el ámbito social, emocional y académico, si bien los niños mejoran con tratamiento, estas dificultades han sido reconocidas como de larga data (Rudolph \& Leonard, 2016).

La heterogeneidad en las características lingüísticas, también se observan en el desempeño lector de los niños con TEL. Así, algunos exhiben dificultades en decodificación y comprensión lectora mientras que otros solo presentan problemas en uno de estos procesos lectores (Kelso, Fletcher, \& Lee, 2007). No obstante, existe consenso al identificar a los niños con TEL como una población de riesgo frente al aprendizaje lector, en comparación a sus pares con DTL (Acosta, Axpe, \& Moreno, 2014; Bishop \& Adams, 1990; Bishop, McDonald, Bird, \& Hayiou-Thomas, 2009; Catts, Bridges, Little, \& Tomblin, 2008; Catts, Fey, Tomblin, \& Zhang, 2002; Coloma et al., 2012; Kelso et al., 2007; Sanz-Torrent, Andreu, Badia, \& Serra, 2010; Snowling, Bishop, \& Stothard, 2000).

Ambos procesos lectores requieren del desarrollo de diferentes habilidades, las que además siguen un curso independiente (J. V. Oakhill, Cain, \& Bryant,
2003). Respecto a la decodificación, se ha establecido su relación con el desarrollo de la conciencia fonológica (McBride-Chang, 1995), con el conocimiento de vocabulario, de letras (Nation \& Snowling, 2004) y con la velocidad de denominación (Catts et al., 2002; Gómez-Velázquez, GonzálezGarrido, Zarabozo, \& Amano, 2010). La conciencia fonológica y el conocimiento de las letras se asocian a la precisión lectora, mientras que la velocidad de denominación lo hace con la fluidez (González, López, Vilar, \& Rodríguez, 2013). Por su parte, los procesos la comprensión lectora se asocian al desarrollo de habilidades de carácter semántico y gramatical. Específicamente, se ha establecido su relación con la capacidad de construir una representación coherente del significado de un texto a partir de procesos de inferenciales, a la capacidad de monitoreo en la comprensión y al dominio de la estructura narrativa (Oakhill \& Cain, 2012).

Los estudios sostienen que en las primeras etapas lectoras la correlación es alta entre decodificación y comprensión lectora, pero una vez que la decodificación se automatiza, esta relación declina en las siguientes etapas escolares (Francis, Fletcher, Catts, \& Tomblin, 2005; Gough, Hoover, \& Peterson, 1996).

Como se mencionó inicialmente, la evidencia no es concluyente respecto a si ambos componentes lectores se encuentran limitados por el TEL. Catts et al. (2008) evaluaron a niños con este diagnóstico desde 2 do a $10 \mathrm{mo}$ grado, reconociendo que presentaban dificultades de rendimiento lector tanto en decodificación como en comprensión lectora. Estas limitaciones fueron más significativas en los primeros años, y durante los siguientes, demostraron 
un crecimiento que fue paralelo al de los niños con DTL, pero siempre se mantuvo inferior hasta el último nivel evaluado. De manera similar, existe evidencia que apoya la hipótesis de "recuperación ilusoria" (Scarborough, 1990) en el proceso evolutivo del TEL y su rendimiento lector (Sanz-Torrent et al., 2010; Snowling et al., 2000). Esta hipótesis sostiene que, si bien los niños se observan con un desempeño lingüístico adecuado, igualmente persistirían con impedimentos que justificarían un rendimiento lector deficitario tanto en decodificación como en comprensión lectora, incluso hasta los 15 años.

Estos antecedentes son coincidentes con el perfil clásico de TEL propuesto por Bishop \& Snowling (2004), a diferencia de otras investigaciones que, si bien advierten limitaciones en comprensión lectora en este déficit, éstas no necesariamente se manifiestan en decodificación. Acorde a ello, Kelso et al. (2007) identificaron un subgrupo de niños con TEL, entre 7,7 y 9,5 años, que presentaban un buen nivel en decodificación, asociado a adecuadas habilidades fonológicas, pero que evidenciaban significativas limitaciones en comprensión lectora. Asimismo, Bishop et al. (2009) agregan evidencia a este hallazgo al identificar niños con TEL entre 9 y 10 años que tampoco manifestaban problemas en la decodificación, asociado a un adecuado desempeño en medidas de conciencia fonológica y en tareas de velocidad de denominación. Sin embargo, presentaban igualmente dificultades de comprensión lectora. Previamente, Bishop \& Adams (1990) ya reportaban resultados semejantes al examinar niños con TEL de 8,6 años promedio, lo que fue coincidente además con una baja comprensión lingüística, reconociendo vínculos débiles entre dificultades fonológicas y rendimiento lector. Cabe destacar, que la presencia de solo dificultades en comprensión lectora en grupos de niños con TEL, también la sugieren estudios con niños hispanohablantes (Acosta et al., 2014; Coloma et al., 2012). Estos impedimentos han sido vinculados a diferentes elementos, como por ejemplo: a un déficit de vocabulario, a una dificultad para recordar y comprender oraciones de distintas estructuras gramaticales (Bishop et al., 2009), a una dificultad para identificar la organización y función de cada palabra dentro de una oración, a limitaciones en los procesos de inferencias (Acosta et al., 2014), a problemas en la identificación de información literal en textos narrativos (Coloma et al., 2012) y a limitaciones en el monitoreo de la comprensión (Oakhill \& Cain, 2012).

Debido a la amplitud de variables posibles que pueden asociarse a estos problemas lectores, la Visión Simple de la Lectura (VSL) propuesta por Hoover \& Gough (1990), surge como un modelo teórico orientador en este debate. La VSL plantea que la comprensión lectora se sustenta tanto por las habilidades de decodificación como por las habilidades de comprensión oral, disociando ambos procesos de comprensión del lenguaje (oral y escrito). A partir de este modelo, es posible identificar tres perfiles de problemas lectores: a) cuando la decodificación es adecuada pero la comprensión lingüística es débil, lo que se ha definido como un déficit específico en comprensión lectora (Acosta et al., 2014), este es el caso de los poor comprehenders, en quienes se observa bajo rendimiento en medidas de comprensión de vocabulario, procesamiento semántico y 
comprensión gramatical (Catts, Adlof, \& Weismer, 2006); b) cuando la comprensión lingüística es adecuada pero la decodificación es débil, como en los casos de dislexia; y c) cuando ambos procesos se encuentran descendidos, condición asociada al perfil clásico de TEL propuesto por Bishop \& Snowling (2004). La descripción de estos perfiles lectores, especialmente el último, permite un acercamiento explicativo a las deficiencias que los niños con este perfil lingüístico pueden presentar. No obstante, los antecedentes expuestos motivan el cuestionamiento frente a la variabilidad en el rendimiento lector en este grupo de niños. Bajo este contexto, se busca aportar evidencia a la caracterización de los problemas lectores en los niños TEL, especialmente en la población de niños hablantes de español porque esta información es escasa. Más aún, dentro de un marco educativo en el que el lenguaje escrito aparece como el principal vehículo de acceso al currículum escolar (Marchant, Lucchini, \& Cuadrado, 2007). Conforme a ello, los objetivos que dirigen este estudio son: a) Comparar los promedios de logro en rendimiento lector entre niños con TEL y DTL que cursan 4to básico; b) Comparar las categorías de desempeños en decodificación y comprensión lectora entre niños con TEL y DTL; c) Identificar el perfil lector de los estudiantes con TEL; y d) Determinar existencia de relación entre ambos procesos lectores en niños de 4to básico con TEL.

\section{Metodología}

\section{Participantes y Criterios de selección}

Las evaluaciones de los estudiantes que conforman la muestra se efectuaron en el marco del
Proyecto FONDECYT Regular № 1130201 que realizó un seguimiento a escolares con TEL en 1ero y 2 do año básico. Luego, mediante el apoyo del Fondo Basal para Centros de Excelencia proyecto FB0003 de PIACONICYT, se evaluaron a los niños también en 4to año utilizando los mismos instrumentos aplicados en 2 do básico.

La muestra total estuvo compuesta por 48 niños de 4to año de educación básica, la que se dividió en dos grupos: TEL y DTL. Ambos fueron conformados por 24 estudiantes distribuidos en 10 mujeres y en 14 hombres cada grupo. La muestra fue tomada de un establecimiento de dependencia particular subvencionado (21 TEL y 19 DTL) y de un establecimiento de dependencia municipal (3 TEL y 5 DTL) de la Región Metropolitana de Santiago de Chile. El promedio de edad de los estudiantes con TEL fue de 10, 3 años y el del grupo control de 9,3 años, si bien los promedios de edad entre ambos grupos difieren en un año, esto no afecta la interpretación de los resultados, debido a que este proceso contempló como criterio el nivel escolar y no el criterio edad. Se obtuvo la autorización de los tutores por medio de un consentimiento informado.

Los escolares con diagnóstico de TEL se seleccionaron a partir del diagnóstico emitido por los fonoaudiólogos pertenecientes a los Programas de Integración Escolar (PIE) de los establecimientos educacionales. Los PIE se rigen bajo el Decreto Supremo 170/2010 (Ministerio de Educación, 2010), en el que se establece un conjunto de criterios para diagnosticar el TEL, que incluyen la valorización de rendimientos en pruebas estandarizadas, junto con el análisis de información obtenida de pautas de evaluación informales. Estos instrumentos miden 
tanto el ámbito comprensivo como el expresivo del lenguaje. Dado el criterio de exclusión que explicita esta normativa para el diagnóstico, se evaluó también audición y habilidades cognitivas no verbales de los estudiantes. Se aplicó una audiometría de barrido de frecuencias 500,1000, 2000 y 4000 Hz, que determinó la audición en rangos normales de los niños con TEL, puesto que sus umbrales auditivos fueron superiores o iguales a $20 \mathrm{~dB}$ (American Speech Language Hearing Association, 2005). Así como también evidenciaron un rendimiento adecuado de habilidades cognitivas no verbales en los resultados del Test de Matrices Progresivas, escala coloreada (Raven, 2005), ya que sus puntuaciones se ubicaron en el percentil 250 sobre este valor, lo que de acuerdo a sus pautas de corrección se interpreta como rendimiento normal. Es importante mencionar que el desarrollo de esta prueba no requiere de respuesta verbal, por lo que las limitaciones lingüísticas del TEL no debiesen haber influido en los resultados.

Para la conformación del grupo control, se estableció como criterio el que los niños cursaran el mismo nivel escolar, que no presentaran antecedentes de asistencia a escuelas de lenguaje, así como tampoco problemas de lenguaje y aprendizaje. Esta información fue brindada por las profesoras de educación básica de los escolares. Igualmente, se corroboró el que sus habilidades cognitivas no verbales y su audición se encontraran en rangos normales.

\section{Instrumentos de evaluación de la lectura}

Para evaluar rendimiento en decodificación se aplicó la subprueba de "Identificación de Letras y Palabras" de la forma revisada en español de la
Batería de Pruebas de Woodcock-Muñoz (Woodcock, Muñoz, Ruef, \& Alvarado, 2005). En esta, se requiere nombrar letras y leer en voz alta una lista de palabras que se presentan en complejidad creciente en términos fonológicos y de frecuencia. Consta de 75 ítems. Cada respuesta correcta es valorada con un 1 punto, por lo que alcanza una puntuación máxima de 75. La prueba presenta una confiabilidad de .96, de acuerdo con la prueba de Kuder y Richardson.

Para evaluar comprensión lectora se utilizaron la subprueba "Comprensión de Textos" (CL1) (Woodcock et al., 2005), y la prueba también llamada "Comprensión de Textos" (CL2) del Test de Lectura y Escritura en Español de (Defior et al., 2006).

La prueba CL1 mide la capacidad de leer comprensivamente palabras, oraciones y textos. En donde inicialmente se deben asociar palabras-dibujos y luego frases-dibujos. Seguidamente se presentan oraciones con distinto grado de complejidad sintáctica que requieren ser completadas con una palabra. Se finaliza con la lectura de textos progresivamente más difíciles por su sintaxis y léxico, los que también deben ser completados con una palabra pertinente al texto. Las respuestas correctas se valorizan con 1 punto y la máxima puntuación es de 32. Presenta una confiabilidad de .95 según la prueba de Kuder y Richardson.

La prueba CL2 busca evaluar la capacidad de relacionar e integrar coherentemente los componentes que integran un texto. Las tareas se desarrollan a partir de dos textos expositivos y uno narrativo. Luego de cada texto, se plantean tres preguntas literales (1 punto por pregunta) y tres de carácter inferencial ( 2 puntos por pregunta). Además, se presenta un ítem de selección de título y otro de 
selección de frase que resuma el texto. En ambos ítems, se asigna 1 punto por elección de idea secundaria y 2 puntos por la elección de la idea principal. La puntuación máxima de la prueba es de 48 puntos. Su confiabilidad es de .69 según el índice alfa Cronbach.

\section{Procedimientos de evaluación}

La aplicación de las tres pruebas descritas se realizó durante el segundo semestre escolar, en el establecimiento de los niños y de forma individual.

En la subprueba "Identificación de Letras y Palabras", se les solicitó a los estudiantes identificar letras entre dibujos, entre otras letras o entre números. Luego, debieron leer palabras que aparecen en un orden de más a menos frecuentes en términos de uso y que además se presentan progresivamente más complejas en su fonología, ortografía y significado.

La aplicación de la subprueba CL1 consistió en solicitar a los niños relacionar un pictograma con una ilustración. Posteriormente, se les pidió indicar la ilustración correspondiente a una frase que debían leer. Finalmente leen oraciones y textos breves que completan con una palabra.

Por último, en la aplicación de la prueba de CL2, se les solicitó a los niños leer cada uno de los textos para luego responder a las preguntas respectivas.

\section{Procesamiento de los datos}

Se obtuvo el puntaje total de las pruebas aplicadas, a partir de ello se calcularon los estadísticos descriptivos de media y desviación típica (DT). La comparación de desempeños se realizó mediante una prueba de muestras independientes.

También se clasificaron en porcentajes los casos del grupo TEL y DTL que obtuvieron desempeños adecuados y descendidos. El punto de corte en las puntuaciones para determinar ambas categorías fue de 1 DT bajo la media del grupo DTL (McArthur, Hogben, Edwards, Heath, \& Mengler, 2000). Este criterio de corte se establece considerando el nivel escolar de los estudiantes (4to básico) y al nivel escolar al que estaban dirigidas las medidas aplicadas (2do básico).

Sumado a ello y bajo estos mismos criterios, se determinó el porcentaje de estudiantes TEL que presentó de forma simultánea dificultades en decodificación y comprensión lectora, así como también el porcentaje de casos que exhibieron dificultades en solo uno de estos procesos lectores. Cabe mencionar que los niños fueron identificados con problemas en comprensión lectora, solo si presentaban rendimientos descendidos (inferior a 1DT) en las dos pruebas que midieron esta habilidad (CL1 y CL2).

Finalmente, se determinó la correlación entre decodificación y comprensión lectora con la Prueba correlación de Pearson. Los análisis estadísticos se efectuaron con el software Statistical Package for the Social Sciences (SPSS) (IBM Corp, 2015) versión 23.

\section{Resultados}

Inicialmente se presenta la comparación de medias de los rendimientos de niños TEL y DTL, tanto en la medida de decodificación como en las dos medidas de comprensión lectora. Se efectúa con la 
prueba $\mathrm{t}$ de Student previamente corroborado el supuesto de homocedasticidad mediante la prueba de Levene. Estos antecedentes se exponen en la Tabla
1. En cada prueba se indican los estadísticos descriptivos de media (M) y de desviación típica (DT).

Tabla 1

Comparación de medias en decodificación y comprensión lectora entre niños con TEL y niños con DTL.

\begin{tabular}{|c|c|c|c|c|c|c|c|}
\hline \multirow[t]{2}{*}{ Desempeños } & \multicolumn{2}{|c|}{$\begin{array}{c}\text { TEL } \\
\text { n } 24 \\
\end{array}$} & \multicolumn{2}{|c|}{$\begin{array}{l}\text { DTL } \\
\text { n } 24 \\
\end{array}$} & \multirow[b]{2}{*}{$\mathrm{t}$} & \multirow[b]{2}{*}{$\mathrm{gl}$} & \multirow[b]{2}{*}{$\mathrm{p}$} \\
\hline & M & D.T. & M & D.T. & & & \\
\hline Decodificación (pje. máx. 75) & 68.87 & 2.75 & 70.16 & 3.01 & 1.54 & 46 & NS \\
\hline CL1 (pje. máx. 32) & 17.29 & 2.23 & 19.70 & 2.92 & 3.21 & 46 & 0.002 \\
\hline CL2 (pje. máx. 48) & 35.91 & 6.85 & 40.45 & 5.02 & 2.61 & 46 & 0.012 \\
\hline
\end{tabular}

$p<0,05 . N S=$ no significativo

Se observa discrepancia de medias en decodificación siendo la del grupo TEL inferior a la del grupo control. Sin embargo, esta diferencia no es estadísticamente significativa. Por el contrario, las medias comparadas de las dos pruebas de comprensión lectora sí difieren significativamente, siendo las del grupo TEL inferiores en ambos casos.

A continuación, se realiza un análisis comparativo de medias de cada uno de los ítems que componen las pruebas CL1 y CL2 en las tablas 2 y 3 respectivamente.

Tabla 2

Comparación de medias ítems prueba CL1 entre niños con TEL y niños con DTL.

\begin{tabular}{|c|c|c|c|c|c|c|c|}
\hline \multirow[t]{2}{*}{ Desempeños CL1 } & \multicolumn{2}{|c|}{$\begin{array}{l}\text { TEL } \\
\text { n } 24\end{array}$} & \multicolumn{2}{|c|}{$\begin{array}{l}\text { DTL } \\
\text { n } 24\end{array}$} & \multirow[b]{2}{*}{$\mathrm{t}$} & \multirow[b]{2}{*}{$\mathrm{gl}$} & \multirow[b]{2}{*}{$\mathrm{p}$} \\
\hline & M & D.T. & M & D.T. & & & \\
\hline 1. Señalar Objetos & \multicolumn{7}{|c|}{ puntuación máxima ambos grupos } \\
\hline 2. Leer frase nominal & 3 & 0.00 & 2.91 & 0.28 & $-1,44$ & 23 & NS \\
\hline 3. Leer y completar oraciones & 9.12 & 1.29 & 9.95 & 1.39 & 2.14 & 46 & 0.03 \\
\hline 4. Leer y completar párrafo expositivo & 2.45 & 1.81 & 3.83 & 1.97 & 2.51 & 46 & 0.01 \\
\hline
\end{tabular}

$p<0,05$. NS=no significativo 
En el ítem 1 la puntuación es máxima en ambos grupos de comparación. En el siguiente se observa desigualdad de las medias, siendo la de TEL levemente superior a la del grupo control, pero ello no representa un peso estadístico. Esta diferencia también se distingue en la comparación de medias de los ítems 3 y 4 , las que sí se interpretan con diferencias significativas siendo las del grupo TEL inferiores en ambos casos.

Tabla 3

Comparación de medias ítems prueba CL2 entre niños con TEL y niños con DTL.

\begin{tabular}{|c|c|c|c|c|c|c|c|}
\hline \multirow[t]{2}{*}{ Desempeños CL2 } & \multicolumn{2}{|c|}{$\begin{array}{c}\text { TEL } \\
\text { n } 24\end{array}$} & \multicolumn{2}{|c|}{$\begin{array}{c}\text { DTL } \\
\text { n } 24\end{array}$} & \multirow[b]{2}{*}{$\mathrm{t}$} & \multirow[b]{2}{*}{ gl } & \multirow[b]{2}{*}{$\mathrm{p}$} \\
\hline & M & D.T. & M & D.T. & & & \\
\hline 1. Preguntas literales & 14.37 & 3 & 15.87 & 1,67 & 2.13 & 36.05 & 0.04 \\
\hline 2. Preguntas inferencial elaborativa & 8.17 & 1.55 & 8.67 & 1.52 & 1.12 & 46 & NS \\
\hline 3. Preguntas inferencial puente & 4.33 & 2.33 & 5.33 & 2.01 & 1.58 & 46 & NS \\
\hline 4. Título & 5.16 & 1.27 & 5.75 & 0.53 & 2.07 & 30.77 & 0.04 \\
\hline 5. Resumen & 3.79 & 1.14 & 4.83 & 1.04 & 3.29 & 46 & 0.00 \\
\hline
\end{tabular}

$p<0,05 . N S=$ no significativo

En todos los ítems de la prueba CL2 se observa que las medias del grupo TEL son inferiores a las del grupo control. Estas desigualdades no representan una diferencia estadística en los ítems 2 y 3, pero sí ocurre en los ítems 1, 4 y 5. En estos últimos, los sujetos deben detectar información literal de los textos, seleccionar el título más pertinente, además de elegir una frase que mejor resuma los textos leídos. Estas dos últimas tareas destacan por requerir la realización de inferencias.

Seguidamente, se presentan los porcentajes de escolares que se situaron en las categorías de desempeños adecuados y descendidos en ambos grupos de estudio. 


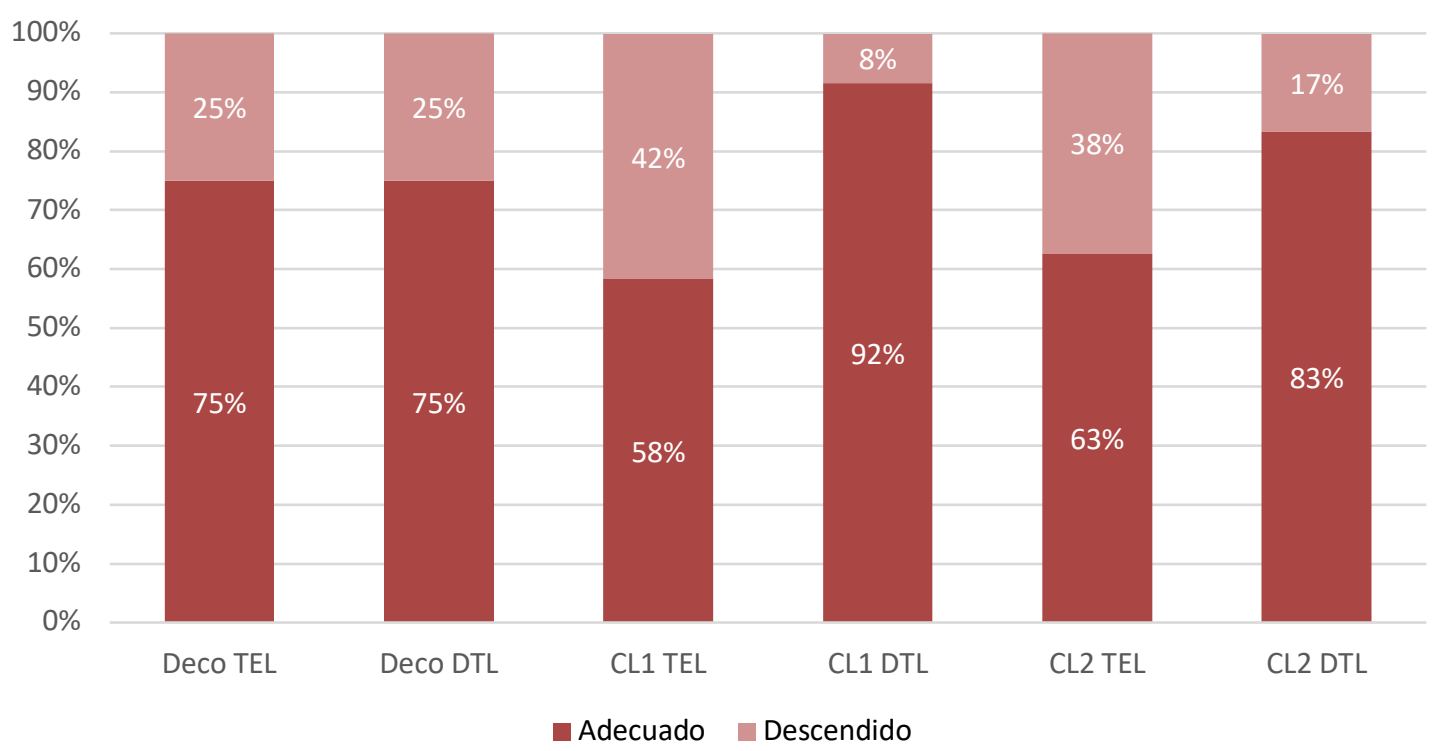

Gráfico 1. Distribución Porcentual de casos de acuerdo con categorías de desempeños en Decodificación - Comprensión Lectora en grupos TEL y DTL

En decodificación, se observa que los dos grupos presentan la misma cantidad de casos en ambas categorías de desempeño. Los porcentajes de rendimientos adecuados y descendidos en este ámbito son del $75 \%$ y $25 \%$ respectivamente.

Respecto a la comprensión lectora, en CL1 la cantidad de niños con TEL que presentan rendimientos adecuados se traducen en un $58 \%$ y los con dificultades en un $42 \%$. En comparación al grupo DTL, destaca que este último valor quintuplica el porcentaje de casos descendidos del grupo control, el que alcanza un $8 \%$ dentro de esta categoría. Cabe señalar además que en esta prueba es donde el grupo
TEL obtiene la mayor cantidad de escolares con rendimientos descendidos, no así el grupo DTL.

Por último, en CL2 TEL alcanza rendimientos adecuados del $63 \%$ y descendidos del $38 \%$. Bajo el mismo análisis, los casos TEL con dificultades superan a más del doble el porcentaje de escolares descendidos del grupo DTL en esta prueba.

Con la finalidad de caracterizar el perfil lector de los escolares con TEL, se individualizó la muestra y se estimó la prevalencia de las dificultades que presentaron en decodificación y en comprensión lectora. 


\section{RENDIMIENTO LECTOR TEL}

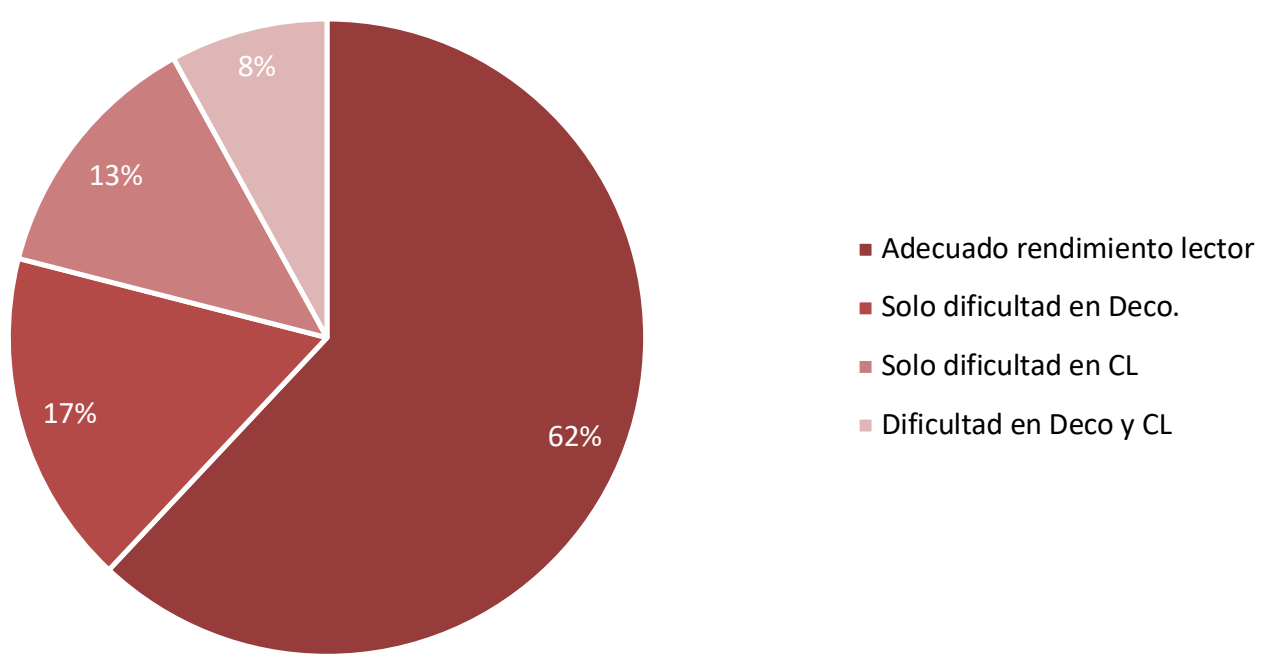

Gráfico 2. Distribución Porcentual del perfil lector de escolares con TEL

Se advierte que el mayor porcentaje de los escolares con TEL no evidencia dificultades lectoras (62\%), el $17 \%$ presenta dificultades solo en decodificación, el 13\% solo en comprensión lectora y el $8 \%$ de los niños exhibe problemas en ambos procesos lectores.

Finalmente, se presenta un análisis de correlación entre decodificación y comprensión lectora con el objetivo de examinar posibles relaciones entre ambos rendimientos lectores. Ello se determina mediante la prueba correlación de Pearson.

Tabla 4

Correlación entre decodificación y medidas de comprensión lectora CL1 y CL2 de niños con TEL y niños con DTL.

\begin{tabular}{lcccc}
\hline & \multicolumn{2}{c}{ TEL } & \multicolumn{2}{c}{ DTL } \\
\cline { 2 - 5 } & $r$ & $p$ & $r$ & $p$ \\
\hline $\begin{array}{l}\text { Decodificación / } \\
\text { CL1 }\end{array}$ & 0.14 & NS & 0.27 & NS \\
$\begin{array}{l}\text { Decodificación / } \\
\text { CL2 }\end{array}$ & 0.09 & NS & 0.41 & 0.04 \\
\hline
\end{tabular}

$p<0,05 . N S=$ no significativo
Se observa que no existe relación entre el rendimiento en decodificación y las dos medidas de comprensión lectora en el grupo TEL. No obstante, en el grupo control sí hubo correlación entre decodificación y CL2, pero esto no ocurrió con CL1. Esta correlación además es de forma positiva.

En síntesis, respondiendo a los objetivos que persigue esta investigación es posible determinar que: el grupo TEL y DTL presentan un comportamiento similar en su rendimiento en decodificación, lo que se traduce en que no presentan diferencias significativas de sus medias en esta área. Ello sí ocurre al comparar las medias de las dos pruebas que miden comprensión lectora, diferencia que asume una significancia estadística y siendo en ambos casos valores inferiores en TEL.

En relación con el análisis detallado de los ítems de estas dos pruebas, es posible concluir que presentan medias inferiores y con diferencias significativas en las tareas de: leer y completar oraciones (CL1); leer y completar textos expositivos (CL1); en la detección de 
información literal (CL2); selección de un título apropiado (CL2); y en la elección de una frase que mejor resuma un texto leído (CL2).

Los resultados previos son coincidentes con que el grupo TEL posee la misma cantidad de casos con rendimientos adecuados y descendidos en decodificación, pero presenta un mayor porcentaje de escolares con desempeños descendidos en ambas pruebas de comprensión lectora en comparación al grupo DTL.

Asimismo, de acuerdo con las categorías de desempeño establecidas en este estudio, se observa un perfil lector variable en el grupo TEL, en el que un alto porcentaje de los escolares no presenta dificultades en desempeño lector, y los que sí lo hacen, exhiben los tres perfiles de dificultades lectoras posibles.

Por último, es posible identificar que en el grupo TEL el rendimiento en decodificación no posee relación con el rendimiento en comprensión lectora, lo que sí es observable parcialmente en el grupo DTL.

\section{Discusión}

Los hallazgos indican que los niños con diagnóstico de TEL presentan promedios de logros significativamente inferiores en comprensión lectora en comparación a los niños con DTL. Sin embargo, exhiben un desempeño conservado en tareas de decodificación. Estos resultados se asemejan a lo documentado por otros estudios (Acosta et al., 2014; D. Bishop \& Adams, 1990; Coloma et al., 2012; Kelso et al., 2007) en los que se han detectado grupos de estudiantes con TEL que solo presentan dificultades en comprensión lectora. En decodificación, los niños con este perfil lingüístico logran leer palabras de diferente metría, complejidad fonológica y frecuencia, de manera similar al grupo DTL. En comprensión lectora, los niños con TEL presentan significativas dificultades al momento de leer y completar tanto oraciones como textos, en la detección de información literal, así como también al momento de seleccionar un título y una frase que mejor resuma un texto. Estas dificultades eran esperables, debido a que se ha identificado que los niños con TEL presentan limitaciones semánticas, sintácticas, al momento de accionar procesos inferenciales (Acosta et al., 2014; Dorothy Bishop et al., 2009) y en la identificación de información literal (Coloma et al., 2012; Sanz-Torrent et al., 2010). No obstante, es importante destacar que el grupo TEL no presentó diferencias significativas con DTL al momento de responder preguntas inferenciales, aunque de todas formas su desempeño en esta tarea fue inferior al del grupo control. Esta situación es similar a los resultados de otro estudio que observó el mismo comportamiento frente a preguntas literales e inferenciales en niños con TEL de 11 años, advirtiendo que ello puede deberse a limitaciones en el uso de la memoria explícita para responder a las primeras, a diferencia de las inferenciales que pueden resolverse mediante el conocimiento del mundo y la pragmática (Sanz-Torrent et al., 2010). Igualmente, otro estudio revela significativas dificultades en la comprensión de preguntas literales a partir de la escucha de textos narrativos en niños con TEL de 6 años, pero no así en las de carácter inferencial (Coloma et al., 2012). Los autores atribuyen esta situación a que las inferencias abordadas fueron de tipo causal y que éstas no representarían mayor dificultad para los niños. De 
manera similar a estos antecedentes, se cree que este comportamiento frente a preguntas inferenciales se debe a que no implicaban la integración del contenido global del texto, sino más bien requerían la realización de inferencias locales. Esta situación explicaría los resultados observados en este ítem, pero es importante precisar que los niños con TEL sí demostraron dificultades significativas en tareas que requerían activar procesos inferenciales a partir de la integración global del texto como, por ejemplo, al momento de seleccionar un título y una frase resumen.

Asimismo, estos promedios significativamente inferiores en las dos pruebas de comprensión lectora son un resultado coherente con el análisis porcentual en el que se reporta una mayor cantidad de escolares con TEL que presentan rendimientos descendidos en esta área en comparación a los niños con DTL. Este análisis de distribución de los casos también refuerza la idea de que ambos grupos presentan un rendimiento similar en decodificación.

Discutidos estos resultados desde el modelo teórico VSL (Hoover \& Gough, 1990), parece factible plantear que los problemas en comprensión lectora se vinculen a dificultades en comprensión oral, debido a que la decodificación se presenta conservada si consideramos que ambos grupos presentan medias similares en este ámbito y que además presentan igualdad de casos adecuados y descendidos en decodificación. Así, el comportamiento del grupo TEL se alejaría al perfil clásico propuesto por Bishop \& Snowling (2004) ajustándose mejor al de déficit específico en comprensión lectora (Acosta et al., 2014). No obstante, se cuestionan estas conclusiones porque además de la comprensión oral y de la decodificación, otros elementos que no son contemplados en la VSL (Hoover \& Gough, 1990) y que no se evaluaron en este estudio, podrían también impactar en la comprensión lectora. Entre estos, la producción sintáctica y léxica (Infante, Coloma, \& Himmel, 2012), la velocidad de denominación (Zevallos, Arcos, \& Ripoll, 2017) o aspectos cognitivos como la memoria de trabajo (Cain, Oakhill, \& Bryant, 2004).

Ahora bien, al individualizar la muestra del grupo TEL con el objetivo de obtener un panorama sobre sus perfiles lectores, destaca que la mayoría de los casos TEL presenta un rendimiento lector adecuado (62\%), seguido en orden decreciente por quienes presentan solo dificultades en decodificación (17\%), solo en comprensión lectora (13\%) y por último, por quienes exhiben problemas en ambos procesos lectores (8\%). El alto porcentaje de niños con TEL sin problemas lectores fue un resultado inesperado debido a que la evidencia plantea que las dificultades lectoras en niños con este déficit lingüístico persisten hasta niveles avanzados de escolaridad (Catts et al., 2008). Estos resultados motivan distintas conclusiones, la primera, da cuenta de una alta variabilidad de los niños con TEL en términos lectores, sumando evidencia a lo que otros estudios también han referido (Bishop et al., 2009; Coloma et al., 2012; Kelso et al., 2007). Si bien menos de la mitad de los casos con TEL presenta dificultades lectoras (38\%), dentro de este porcentaje inferior se observa esta cualidad. Más aún, desde este nuevo análisis podría ser posible identificar dentro de este grupo, la presencia de los tres tipos de problemas lectores propuestos por la VSL lo que, de alguna forma, tensiona el postulado inicial de esta discusión que 
indica mayor ajuste al perfil del déficit específico en comprensión lectora. En esta misma línea, impresiona en este nuevo análisis la presencia de mayor cantidad de casos TEL con problemas solo en decodificación, lo que a primera vista parece contradictorio a los resultados iniciales, no obstante, esto tiene relación con una cuestión metodológica. Se determinó con problemas de comprensión lectora, aquellos casos que muestran rendimientos descendidos (inferior a 1DT) en ambas pruebas que miden esta habilidad (CL1 y CL2). Así, estudiantes con desempeños descendidos en decodificación y en una de las pruebas de comprensión lectora, fueron identificados con problemas solo en decodificación. Igualmente, otros niños obtuvieron rendimiento descendido solo en una de las pruebas de comprensión lectora junto con adecuado desempeño en decodificación, y estos casos conformaron cerca de la mitad del porcentaje de niños que se reportan con adecuado rendimiento lector.

Otra conclusión sugerente respecto a la cantidad de niños sin problemas lectores es que hayan superado estas barreras producto de la intervención recibida de los PIE dentro de sus escuelas durante sus cuatro años de escolarización. Indudablemente esta premisa requiere de antecedentes de corte longitudinal que aporten información a la elaboración de esta tesis. Otro elemento para considerar es que este estudio utilizó medidas dirigidas a escolares de 2 do básico y esto también pudo haber incidido en que se presente un alto porcentaje de casos TEL sin problemas lectores.

En base a este último dato, se esperaba que todos los escolares del grupo DTL alcanzaran desempeños adecuados en las evaluaciones. No obstante, DTL presentó un $29 \%$ de casos con rendimientos descendidos. Destaca que la mayoría de estos casos se relacione con dificultades en decodificación, porque el supuesto es que este aprendizaje se encuentre logrado en 4to básico, al igual que comprensión lectora de acuerdo con lo que establece el curriculum escolar chileno. Sin embargo, otros estudios ya han reportado resultados similares en niños de este mismo nivel escolar y que pertenecen a escuelas públicas chilenas (Infante, 2006; Infante et al., 2012). Bajo este contexto, es pertinente destacar que los escolares de la muestra pertenecen a establecimientos de dependencia municipal (públicos) y particular subvencionada. Antecedentes que pueden favorecer la comprensión a este fenómeno, es que los primeros, si bien han demostrado un avance significativo en la última década en los resultados del Sistema de Evaluación de la Calidad de la Evaluación (SIMCE) Comprensión de Lectura de 4to básico, aun así, presentan una diferencia negativa de más de 60 puntos con los rendimientos de las escuelas particulares pagadas (Ministerio de Educación, 2016); los segundos por su parte, muestran mínimas diferencias en sus desempeños si es que se controla el factor socioeconómico, por lo que no representarían un mejor escenario en términos académicos (Castillo, González, \& Puga, 2011; Ministerio de Educación, 2016). En base a estos antecedentes y a partir de los hallazgos de este estudio, se hipotetiza que estas dificultades puedan asociarse a problemas para decodificar incluso en este nivel escolar. Otro aspecto que puede aportar a la comprensión del rendimiento descendido en el grupo DTL, es que CL1 y CL2 contemplan el uso del texto expositivo. Al respecto, 
se ha identificado que los estudiantes presentan escaso acceso a este tipo de estructuras en la escuela, a pesar de que surge como una competencia clave para el éxito académico y también laboral (Meyer \& Ray, 2011). La baja exposición a este tipo de textos posee un impacto negativo dado que el discurso expositivo o informativo requiere de una enseñanza formal explicita, pues no forma parte del acto comunicativo cotidiano en los niños, a diferencia de la conversación o narración. Este último se construye en torno a los eventos, mientras que el texto expositivo se conforma en a partir de un tópico, por lo que las relaciones que se establecen dentro de estos discursos son distintas (Concha, Aravena, Coloma, \& Romero, 2010).

Por otra parte, se identificó ausencia de correlación entre ambos procesos en el grupo TEL. Esto puede deberse a que esta relación sea observable solo si es que ambos procesos se presentan adecuados, tal cual ha sido sugerido por otros estudios (Coloma et al., 2012; Infante \& Coloma, 2005). Bajo esta premisa, la inexistencia de correlación en TEL se debería a sus rendimientos deficientes en comprensión lectora. Aunque esta tesis es discutible, puesto que un estudio que utilizó las mismas subpruebas de Woodcock et al. (2005) para evaluar desempeño lector, sí halló correlación entre ambos procesos, pero en una muestra de escolares con TEL de 1ero básico (6,8 años) que exhibieron dificultades tanto en decodificación como en comprensión lectora (Coloma, Sotomayor, De Barbieri, \& Silva, 2015). Ello podría sugerir que la factibilidad de la correlación esté dada por la igualdad en el rendimiento de ambos procesos lectores, independiente si las puntuaciones alcanzadas sean altas o bajas.

En cuanto al grupo DTL, se esperaba una baja o moderada correlación entre decodificación y comprensión lectora porque los escolares se encuentran cursando 4to año de educación básica, que corresponde al último nivel del primer ciclo de la educación escolar chilena, y está referido que esta correlación pierde fuerza a medida que se avanza de nivel escolar (Francis et al., 2005). No obstante, esta espectativa inicial se cumplió pero parcialmente, debido a que el grupo DTL presentó esta correlación y de forma moderada (sig. $0.04 * p<0.05$ ), pero solo con CL2. Así, al presentar mayor porcentaje de puntuaciones en esta prueba (84\%) en comparación a CL1 (62\%), sí logra establecer correlación entre ambos procesos, tal cual lo han señalado otros estudios al evaluar niños con DTL (Balbi, Cuadro, \& Trías, 2009; Infante \& Coloma, 2005; Infante et al., 2012; Zevallos et al., 2017). Este antecedente sumaria evidencia de apoyo a la hipótesis inicial sobre la necesidad de que ambos procesos lectores se presenten adecuados para presentar correlación.

Se concluye que los niños evaluados con diagnóstico de TEL y que cursan 4to básico, son un grupo de riesgo frente al aprendizaje lector, porque exhiben significativas dificultades en comprensión lectora cuando el análisis se realiza en base a las medias de sus rendimientos. Estas no se explican por sus desempeños en decodificación, porque TEL se comporta de forma similar al grupo control en esta medida. Igualmente, se cree que este estudio aporta a evidenciar la heterogeneidad del rendimiento lector en este déficit lingüístico, en el que incluso un porcentaje importante de casos no da cuenta de problemas lectores. 
Cabe mencionar que este estudio admite limitaciones metodológicas. Por un lado, para precisar posibles variables asociadas a las dificultades en comprensión lectora, debido a que no midió elementos claves para esta discusión como por ejemplo la comprensión oral. Precisar el rendimiento en comprensión oral, podría aportar a reconocer si a pesar de la variabilidad lectora evidenciada, aun así, los escolares con TEL comparten características lingüísticas. Por otra parte, contar con antecedentes longitudinales contribuiría a esclarecer si los perfiles lectores observados en este estudio, corresponden a características que los escolares ya presentaban en los niveles iniciales, o si más bien se asocian a un perfil nuevo generado por cambios evolutivos productos del proceso escolar aportando evidencia clave para los programas de intervención.

Agradecimiento: se agradece el apoyo de Carmen Julia Coloma Tirapegui durante todo el desarrollo de este estudio.

Patrocinio: estos estudios fueron financiados por CONICYT - PCHA/Magíster Nacional/ 2015-folio.

\section{Referencias}

Acosta, V., Axpe, M. de los Á., \& Moreno, A. M. (2014). Rendimiento lingüístico y procesos lectores en alumnado con Trastorno Específico del Lenguaje. Revista Española de Pedagogía, 72(259), 477-490.

American Speech Language Hearing Association. (2005). Audiology Information Series. ASHA.

Balbi, A., Cuadro, A., \& Trías, D. (2009). Comprensión lectora y reconocimiento de palabras. Ciencias Psicológicas, 3(2), 153-160.
Bishop, D., \& Adams, C. (1990). A prospective study of the relationship between specific language impairment, phonological disorders and reading retardation. Journal of Child Psychology and Psychiatry, and Allied Disciplines, 31(7), 1027-1050.

Bishop, D., McDonald, D., Bird, S., \& Hayiou-Thomas, M. (2009). Children who read words accurately despite language impairment: who are they and how do they do it? Child Development, 80(2), 593-605. https://doi.org/10.1111/j.1467-8624.2009.01281.x

Bishop, D., \& Snowling, M. (2004). Developmental dyslexia and specific language impairment: same or different? Psychological Bulletin, 130(6), 858-886. https://doi.org/10.1037/0033-2909.130.6.858

Cain, K., Oakhill, J., \& Bryant, P. (2004). Children's Reading Comprehension Ability: Concurrent Prediction by Working Memory, Verbal Ability, and Component Skills. Journal of Educational Psychology, 96(1), 31-42.

Castillo, P., González, A., \& Puga, I. (2011). Gestión y efectividad en educación: evidencias comparativas entre establecimientos municipales y particulares subvencionados. Estudios pedagógicos (Valdivia), 37(1), 187-206. 07052011000100010

Catts, H., Adlof, S., \& Weismer, S. (2006). Language deficits in poor comprehenders: a case for the simple view of reading. Journal of Speech, Language, and Hearing Research: JSLHR, 49(2), 278-293. https://doi.org/10.1044/1092-4388(2006/023)

Catts, H., Bridges, M., Little, T., \& Tomblin, J. B. (2008). Reading achievement growth in children with language impairments. Journal of Speech, Language, and Hearing Research: JSLHR, 51(6), 1569-1579. https://doi.org/10.1044/1092-4388(2008/07-0259)

Catts, H., Fey, M., Tomblin, J. B., \& Zhang, X. (2002). A longitudinal investigation of reading outcomes in children with language impairments. Journal of Speech, Language, and Hearing Research: JSLHR, 45(6), 1142-1157.

Coloma, C. J., Pavez, M. M., Peñaloza, C., Araya, C., Maggiolo, M., \& Palma, S. (2012). Desempeño lector y narrativo en escolares con trastorno específico del lenguaje. Onomázein, 26(2), 351-375.

Coloma, C. J., Sotomayor, C., De Barbieri, Z., \& Silva, M. (2015). Comprensión lectora, habilidades lingüísticas y decodificación en escolares con trastorno específico del lenguaje. Revista de Investigación en Logopedia, 5(1), 1-17. 
Concha, S., Aravena, S., Coloma, C. J., \& Romero, V. (2010). Escritura expositiva en tres niveles de escolaridad: coherencia y dominio de recursos lingüísticos. Literatura y lingüística, (21), 75-92. https://doi.org/10.4067/S071658112010000100007

Defior, S., Fonseca, L., Gottheil, B., Aldrey, A., Pujals, M., Rosa, G., ... Serrano, F. (2006). LEE - Test de lectura y escritura en español. Buenos Aires: Paidós. Recuperado de http://www.leetestdelecturayescritura.com/

Francis, D., Fletcher, J., Catts, H., \& Tomblin, J. B. (2005). Dimensions affecting the assessment of reading comprehension. En S. Paris \& S. Stahl (Eds.), Children's Reading Comprehension and Assessment (1. ${ }^{\mathrm{a}}$ ed., pp. 369394). Mahwah, N.J: Erlbaum.

Gómez-Velázquez, F., González-Garrido, A., Zarabozo, D., \& Amano, M. (2010). La velocidad de denominación de letras: el mejor predictor temprano del desarrollo lector en español. Revista Mexicana de Investigación Educativa, 15(46), 823-847.

González, R. M., López, S., Vilar, J., \& Rodríguez, A. (2013). Estudio de los predictores de la lectura. Revista de Investigación en Educación, 11(2), 98-110.

Gough, P., Hoover, W., \& Peterson, C. (1996). Some observations on a simple view of reading. En C. Cornoldi \& J. V. Oakhill (Eds.), Reading Comprehension Difficulties: Processes and Intervention (1. ${ }^{\mathrm{a}}$ ed., pp. 1-13). Mahwah, N.J: Lawrence Erlbaum Associates.

Hoover, W. A., \& Gough, P. B. (1990). The simple view of reading. Reading and Writing, 2(2), 127-160. https://doi.org/10.1007/BF00401799

IBM Corp. (2015). IBM SPSS Statistics (Versión 23). Armonk, NY: IBM Corp.

Infante, M. (2006). Las dificultades específicas de aprendizaje en lectura y su relación con habilidades lingüísticas en contextos socioeconómicos diversos. Revista Chilena de Fonoaudiología, 7(1), 29-44. https://doi.org/10.5354/0719-4692.2006.48360

Infante, M., \& Coloma, C. J. (2005). Comprensión oral y lectura: ¿qué sucede cuando la decodificación ha sido superada? Boletín de Investigación Educacional, 20(2), 267280.

Infante, M., Coloma, C. J., \& Himmel, E. (2012). Comprensión lectora, comprensión oral y decodificación en escolares de $2^{\circ}$ y $4^{\circ}$ básico de escuelas municipales. Estudios Pedagógicos, 38(1), 149-160. https://doi.org/10.4067/S0718-07052012000100009
Kelso, K., Fletcher, J., \& Lee, P. (2007). Reading comprehension in children with specific language impairment: an examination of two subgroups. International Journal of Language \& Communication Disorders, 42(1), 39-57. https://doi.org/10.1080/13682820600693013

Marchant, T., Lucchini, G., \& Cuadrado, B. (2007). ¿'Por qué Leer Bien es Importante?: Asociación del Dominio Lector con Otros Aprendizajes. Psykhe (Santiago), 16(2), 3-16. https://doi.org/10.4067/S0718-22282007000200001

McArthur, G. M., Hogben, J. H., Edwards, V. T., Heath, S. M., \& Mengler, E. D. (2000). On the "specifics» of specific reading disability and specific language impairment. Journal of Child Psychology and Psychiatry, and Allied Disciplines, 41(7), 869-874.

McBride-Chang, C. (1995). What is phonological awareness? Journal of Educational Psychology, 87(2), 179-192. https://doi.org/10.1037/0022-0663.87.2.179

Meyer, B., \& Ray, M. (2011). Structure strategy interventions: Increasing reading comprehension of expository text. International Electronic Journal of Elementary Education, 4(1), 127-152.

Ministerio de Educación. Decreto Supremo 170 (2010). Recuperado

https://www.leychile.cl/Navegar?idNorma=1012570

Ministerio de Educación. (2016). Resultado Educativos 2016 (Informe) (p. 98). Chile. Recuperado de http://archivos.agenciaeducacion.cl/ResultadosNacionales 2016_.pdf

Nation, K., \& Snowling, M. J. (2004). Beyond phonological skills: broader language skills contribute to the development of reading. Journal of Research in Reading, 27(4), 342-356. https://doi.org/10.1111/j.14679817.2004.00238.x

Oakhill, J., \& Cain, K. (2012). The Precursors of Reading Ability in Young Readers: Evidence From a Four-Year Longitudinal Study. Scientific Studies of Reading, 16(2), 91121. https://doi.org/10.1080/10888438.2010.529219

Oakhill, J. V., Cain, K., \& Bryant, P. E. (2003). The dissociation of word reading and text comprehension: Evidence from component skills. Language and Cognitive Processes, 18(4), 443-468. https://doi.org/10.1080/01690960344000008

Raven, J. C. (2005). Test de matrices progresivas. Escala coloreada, general y avanzada. Buenos Aires: Paidós. 
Rudolph, J., \& Leonard, L. (2016). Early Language Milestones and Specific Language Impairment. Journal of Early Intervention, 38(1), 41-58.

https://doi.org/10.1177/1053815116633861

Sanz-Torrent, M., Andreu, L., Badia, I., \& Serra, M. (2010). EI proceso lector en niños con antecedentes de retraso y trastorno específico del lenguaje. Revista de Logopedia, Foniatría y Audiología, 30(1), 23-33. https://doi.org/10.1016/S0214-4603(10)70005-X

Scarborough, H. S. (1990). Very early language deficits in dyslexic children. Child Development, 61(6), 1728-1743.

Snowling, M., Bishop, D. V., \& Stothard, S. E. (2000). Is preschool language impairment a risk factor for dyslexia in adolescence? Journal of Child Psychology and Psychiatry, and Allied Disciplines, 41(5), 587-600.

Woodcock, R., Muñoz, A., Ruef, M., \& Alvarado, C. (2005). Language survey-revised. Test Book- Spanish. Rolling Meadows: Riverside Publishing Company.

Zevallos, D., Arcos, N., \& Ripoll, J. (2017). La concepción simple de la lectura en alumnos de $4^{\circ}$ de primaria de Quito. Alteridad, 12(1), 115-122. 